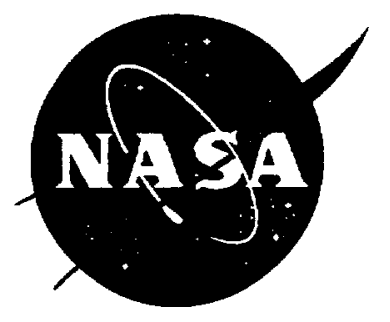

Particle Acceleration by CME-Driven Shock Waves

Donald V. Reames

Code 661, Laboratory for High Energy Astrophysics

NASA Goddard Space Flight Center

Greenbelt, MD 20771

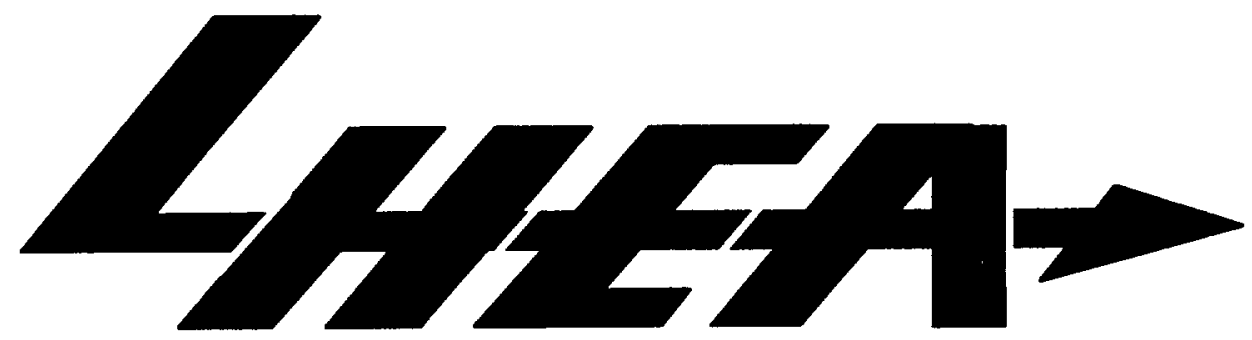

National Aeronautics and Space Administration Goddard Space Flight Center Greenbelt, Maryland 20771 


\title{
Particle Acceleration by CME-Driven Shock Waves
}

\author{
Donald V. Reames \\ NASA/ Goddard Space Flight Center, Greenbelt, MD 20771
}

Highlight Paper, presented at the $26^{\text {th }}$ International Cosmic Ray Conference Salt Lake City, UT

August 17-25, 1999 


\title{
Particle Acceleration by CME-Driven Shock Waves
}

\author{
Donald V. Reames \\ NASA/ Goddurd Space Flight Center, Greenbelt, MD 20771
}

\begin{abstract}
In the largest solar energetic particle (SEP) events, acceleration occurs at shock waves driven out from the Sun hy coronal mass ejections (CMEs). Peak particle intensities are a strong function of CME speed. although the intensities, spectra, and angular distributions of particles escaping the shock are highly modified by scattering on Alfven waves produced by the streaming particles themselves. Element abundances vary in complex ways hecause ions with different values of $Q / A$ resonate with different parts of the wave spectrum, which varies with space and time. Just recently, we have begun to model these systematic variations theoretically and to explore other consequences of proton-generated waves.
\end{abstract}

\section{INTRODUCTION}

Before the discovery of CMEs, it was believed that all SEPs were accelerated in solar flares. This left serious questions of how the particles could cross from one magnetic field line to another to yield high intensities over $\sim 180^{\circ}$ of solar longitude and why events lasted for days when the flares themselves were over in a few hours at most. With the observation of CMEs and improving observations of SEPs, it became clear that the largest "gradual" proton events were associated with CME driven shock waves (11). At the highest energies in the largest events, measurements of the mean charge state of Fe found that $\mathrm{Q}_{\mathrm{Fe}} \sim 14$ at energies out to $6(00 \mathrm{MeV} / \mathrm{amu}$ (46). This showed that these ions came from ambient coronal temperatures and not from flare-heated material. Furthermore. these ions would be rapidly stripped at coronal densities (40) and were probably accelerated beyond 2 solar radii. A distance of $>5$ solar radii was independently derived by Kahler (10) for peak acceleration of $1-21 \mathrm{GeV}$ protons observed in ground-level events.

Meanwhile, SEPs that do come from impulsive flares are the smaller " $\mathrm{He}$-rich" events $(27,32)$ where $Q_{1 c} \sim 20(17)$. The unique 1000 -fold enhancement of ${ }^{3} \mathrm{He} / \mathrm{He}$ in these events is believed to come from resonant wave-particle interactions in the flare plasma (e.g. 39).

Most physicists practicing today learned the origin of SEP events from their research advisers in simpler times when the "solar flare myth" (7) prevailed. Perhaps because research advisors are often as revered as gods, the idea seems to have lodged as a religious belief that is difficult to shake, and shock acceleration is still viewed as heresy in some quarters. However. those willing to tempt fate will find that a consistent 
picture of distinct "gradual" and "impulsive" events emerges that spans a wide range of observations from photons to ionization states 10 abundances and spectra from $20 \mathrm{keV}$ $1020 \mathrm{GeV}$.

A complete comparison of the properties of gradual and impulsive SEP events and their sources is beyond the scope of this paper (see reviews 27, 29, 30, 32, 14, 15. 9. 7). Recent multi-spacectaft studies of the spatial distribution of SEPs around the CMI: and shock have been reported by Reames, Barbier. \& $\mathrm{Ng}$ (33) and by Reames, Kahler, \& Ng (34). These include the phenomenon of spatially and temporally invariant spectra produced when particles are quasi-trapped in expanding magnetic hottles formed by CME loops or behind the shock (see also 32 ).

This paper seeks only to highlight certain features of SEP events. It is not a review. For a more comprehensive review of both gradual and impulsive SEP events and their relationship to other heliospheric acceleration sources, see the recent review by Reames (32). In the present paper, we consider two recent updates on the properties of CMEassociated SEP events. energy-dependent variations in ionization states and correlations between SEP intensities and CME speeds. We then discuss numerous consequences of wave-particle interactions near shocks and finally consider the high-energy "knee" of the spectrum where shock acceleration efficiencies plummet.

\section{IONIZATION STATES}

One of the distinctive signatures of shock acceleration has been the ionization states of the elements from 0.3 to $600 \mathrm{MeV} / \mathrm{amu}$. especially Fe, that are similar to those of the solar wind. Not only do these ionization states represent ambient, unheated coronal plasma, but, in addition, the ions must be accelerated at high ahitudes above the corona where densities are too low for stripping to occur.

Figure 1 shows new measurements of ionization states in the event of 1997 November $6(18,20)$. These indicate strong energy dependence in just the region where the ion speed begins to exceed the thermal electron speed. If shock acceleration begins low enough in the corona that the density is $\sim 10^{9}$ $\mathrm{cm}^{-3}$, ions might come to an equilibrium charge state appropriate to their speed relative to the plasma electrons. A simple extension of the common expression for equilib-

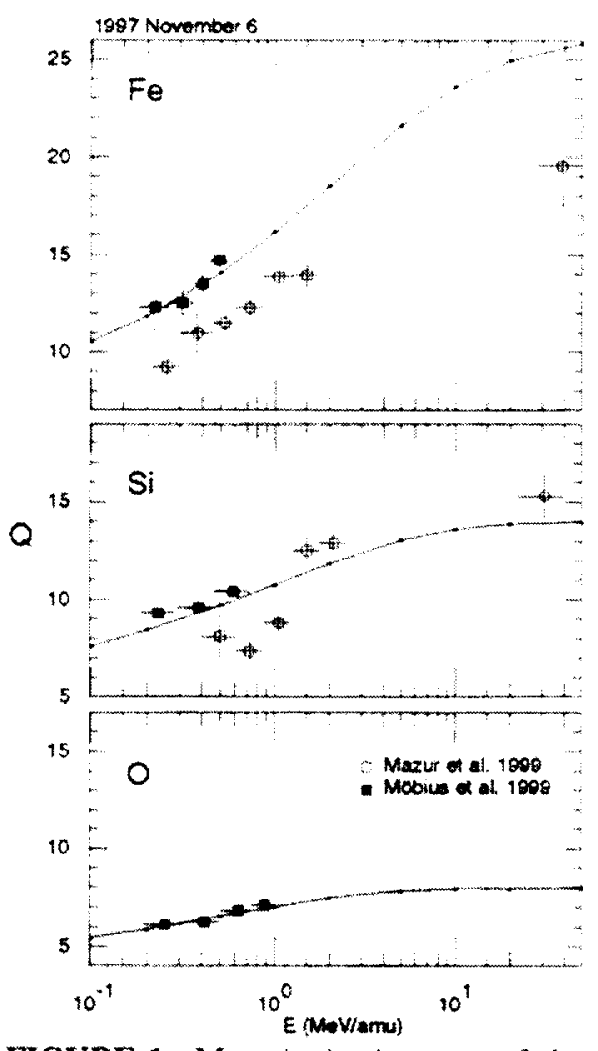

FIGURE 1. Mean ionization state of elements in the 1997 November 6 SEP event (18, 20) are compared with a simple theory of stripping during shock acceleration in the upper corona (38). 
rium charge states (38) is compared with the data in Figure 1 . The measured Fe charge at 4) $\mathrm{MeV} / \mathrm{amu}$ falls below the equilibrium curve, perhaps indicating either that le has not traversed enough material to attain equilibrium or that acceleration cont inued after the shock left the corona. or both.

Within the present accuracy of the measurements. the energy dependence of the charge states in this large SEP event is consistent with shock acceleration in the low corona. At low energies, when the ion speed is less than the thermal electron speed. ionization states still define a plasma temperature that is consistent with an ambient coronal temperature of $2 \mathrm{MK}$.

One should be aware of recent papers, such as that of Barghouty and Mewaldt (1), that completely neglect ion speed and determine ionization states using the plasma temperature alone, even for ion energies up to $-100 \mathrm{MeV} / \mathrm{amu}$. This paper leaves the erroneous impression that shocks are incapable of producing highly ionized ions.

\section{CORRELATIONS OF PEAK INTENSITIES AND CME SPEEDS}

Another strong indication of the origin of particles in the large SEP events is the correlation between peak proton intensities and maximum CME speed that was originally observed by Kahler et al. (11). We have recently reexamined this correlat ion for two different samples of events (35). The first list of events was used in a recent ISTP workshop with CME speeds determined by the SOHO/LASCO coronagraph. and particle intensities measured on the Wind and IMP- 8 spacecraft. The second is an older list of events using the SOL.WIND coronagraph at Earth while the Helios spacecraft observed protons off the solar limbs. Both lists were prepared to look at correlations of CMEs and interplanetary shock waves, without regard for the presence or absence of energetic particles. Peak proton intensities. or upper limits. were determined at -2 and $-20 \mathrm{MeV}$ for these events and their correlation with CME speed is shown in Figure 2.

The correlation coefficients $(r=0.62$ and 0.72 ) listed in Figure 2 are quite high compared with corresponding correlations $(\mathrm{I} \sim 0.3$ ) of particle intensities with flare properties such as X-ray or $\gamma$-ray intensities. In fact, the correlation with CME speed is surprisingly high, considering that many factors are not considered, such as the longitude of the event, the CME speed on the observers actual field line, the speed of the material upstream of the shock, and the angle between the magnetic field and the shock normal and its time evolution.

Because of the steep dependence of particle intensities on CME speed, only the fastest 1-2\% of all CMEs drive fast enough shock waves to accelerate any particles at all. Most CMEs are emitted with speeds that barely exceed that of the solar wind. This speed correlation strongly suggests that it is the shock ahead of the CME where acceleration occurs, rather than the reconnection region behind it, for example. The existence of many large. slow CMEs ( $-98 \%$ of them) with a complete absence of energetic particles further supports this conclusion. 


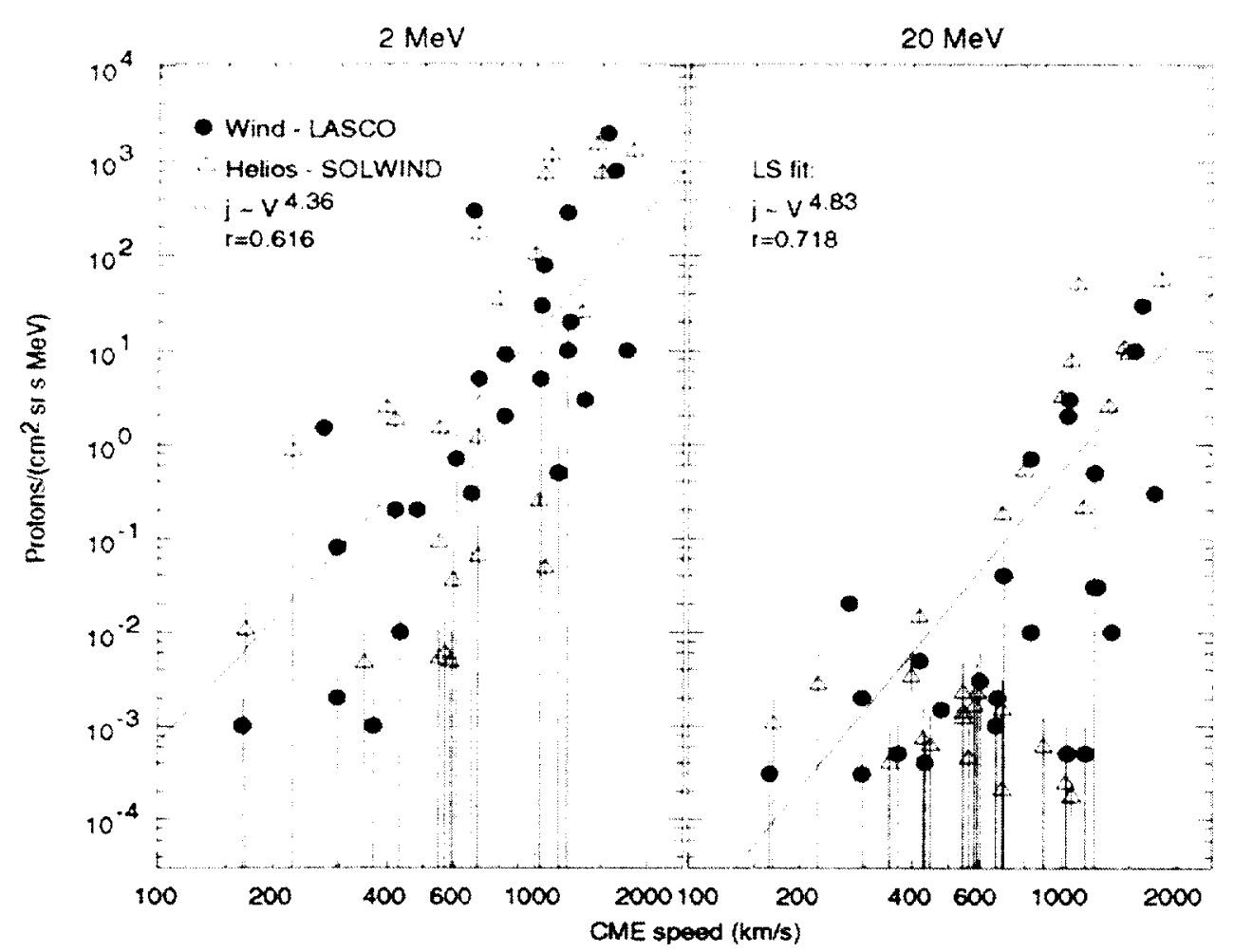

FIGURE 2. Correlations of peak proton intensities at -2 and $\sim 20 \mathrm{MeV}$ with CME speed are shown for 2 different event lists. Correlation coefficients are relatively high and IS fit lines show a steep dependence of the intensities on speed (35).

\section{PROTON GENERATED WAVES}

It is a fundamental fact of plasma physics that protons streaming along magnetic fields can generate or amplify Alfven waves (41). These waves modify the transport of particles that come behind. Different particle species at the same velocity resonate with different regions of the wave spectrum because of their different values of charge-tomass ratio, $Q / A$. Hence, element abundances can vary in space and time in response to the changing wave spectrum. The role of self-generated waves in acceleration at interplanetary shocks was first considered in detail by Lee (13). These waves produce what is referred to nowadays as a "cosmic-ray modified" shock.

Ironically, waves that can scatter particles are notoriously difficult to resolve from the background turbulence by direct measurements. Fisk (6) pointed out long ago that particle transport is grossly inconsistent with observed magnetic power spectra. Presumably this occurs because much of the variation lies in a direction orthogonal to the magnetic field and is not seen by the particles traveling along the field (2). However, the enhanced spectra of self-generated wave near shocks. where streaming is largest, have been found to be consistent with the expectations of Lee (13) theory (12.42.43. 49). Of course. the newly-generated wave energy at a fast shock is less than a few percent of the energy in SEPs. which is smaller than the magnetic energy. 


\section{Streaming-Limited Intensities}

Figure 3(a) shows superposed intensity-time profiles of several events observed on Helios (26). Early in these events proton intensities do not exeeed $-200\left(\mathrm{~cm}^{2} \mathrm{sr} s\right.$ $\mathrm{MeV}^{-2}$, although later they may rise 1 or 2 orders of magnitude at the time of shock passage. There are no events near $1 \wedge \mathrm{U}$ with significantly higher intensities of $\mathrm{MeV}$ protons early in the event. $\mathrm{Ng}$ and Reames (23) showed theoretically that these intensities were limited by self-generated waves produced at a shock near the Sun when streaming protons produced sufficient wave intensities to throttle the streaming. In sufficiently large events, higher-energy particles are also streaming-limited. but at lower intensities as shown in Figure 3(b). Here again, peak intensities occur at the time of shock passage, even at 100-500 MeV. Recently, Reames and $\mathrm{Ng}$ (37) undertook a statistical study of the streaming limit, its energy dependence and its radial gradient.

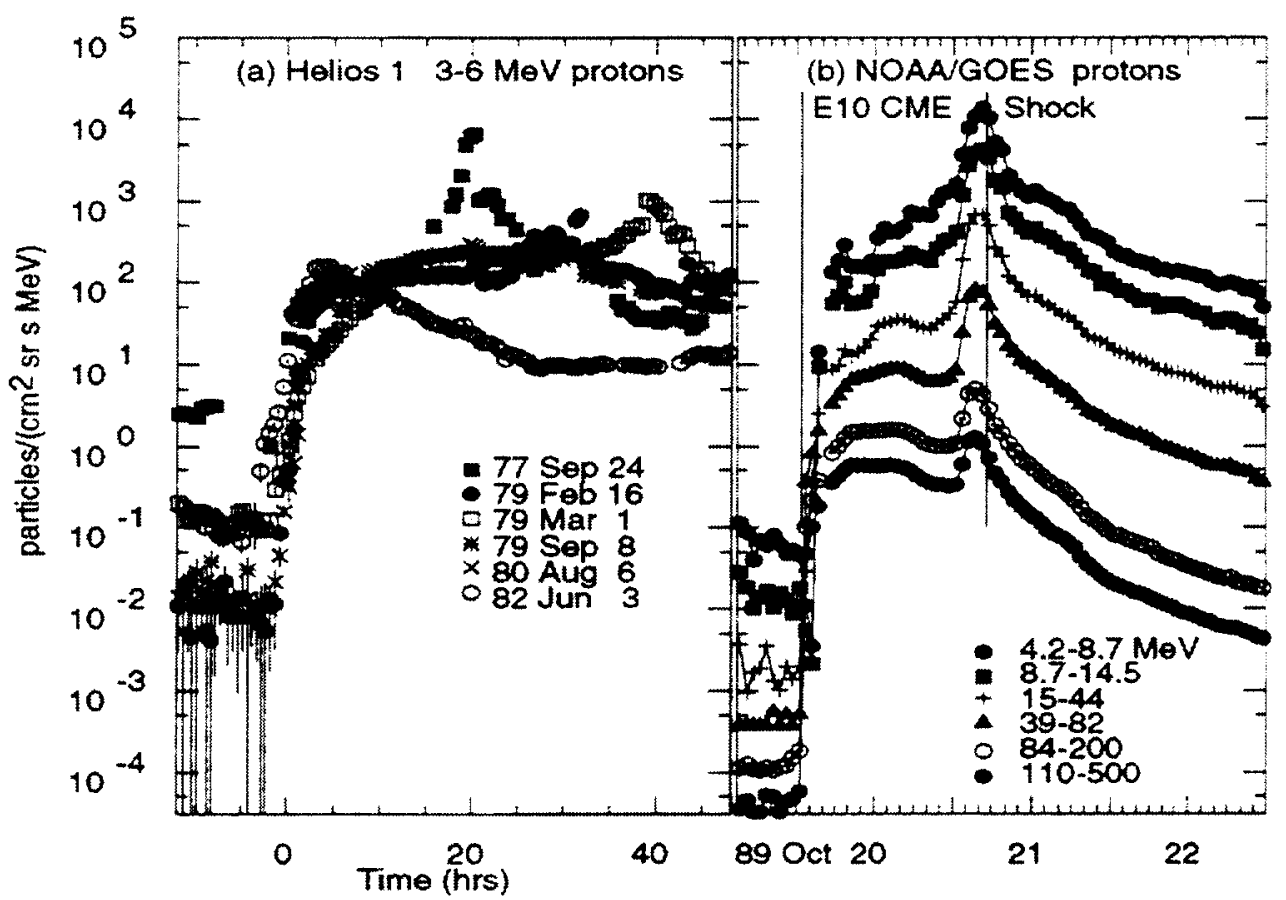

FTGURE 3. Panel (a) shows superposed intensity-time profiles of 3-6 MeV protons in several events with streaming-limited intensities early in the events. Panel (b) shows similar limits as a function of energy in the large 1989 October 19 event. Note that intensities often peak at the time of shock passage (32).

\section{Abundance Variations}

Measurements of energetic particles at a few MeV/amu have been used to determine element abundances in the solar corona for many years $(22,3.28,31,32)$. When the element abundances are averaged over many events, are divided by the corresponding photospheric abundances, and are plotted as a function of the first ionization potential (FIP), one obtains the distinctive plot that is believed to describe the ion-neutral fractionation during formation of the solar corona. 
In dealing with event-to-event variations. workers in this field have historically considered all large events. Fe-rich to Fe-poor. either ranking them by their $\mathrm{Fc} / \mathrm{O}$ ratios (22) or averaging over all the events $(3,28,31)$. Breneman and Stone (3) found that element abundances in an event had a rough power-law dependence on $Q / A$ of the elements, both for Fe-rich and Fe-poor events. They were able to rank -20 elements in this way, and to determine coronal abundances for each. Had Breneman and Stone used modern photospheric abundances for their comparisons, they would have found that the abundances, averaged over all of their events. could be directly interpreted as "coronal" abundances, without correction.

However, two elements that failed to fit the $Q / A$ dependence were simply omitted by Breneman and Stone. namely $H$ and $H$. Surely, one must be wary of a model that fails to include the dominant elements of the plasma. The elements $\mathrm{C}$ through $\mathrm{Zn}$ correlate well with $\mathrm{Fe}$; based on 49 large SEP events, for example, Si/O vs. Fe/O gives a correlation coefficient of $0.90(28)$. However, $\mathrm{He} / \mathrm{H}$ vs. Fe/O gives a coefficient of only 0.31 (28). Plots of abundances vs. $Q / A$ show disorganized behavior when $\mathrm{H}$ and He are included (32). One can hardly blame this behavior on an uncertainty about the ionization state of $\mathrm{H}$. Our discipline has harbored this secret about $\mathrm{H}$ and $\mathrm{He}$ for many years. Yet, things would only get worse before they got better.

For many years there had been some evidence for time dependence of abundances during an event, but we were unprepared for the smooth systematic variations we began to see with the large-geometry instruments on the Wind spacecraft. These variations are seen in Figure 4, where the abundances in the lower panel are normalized to coronal abundances (see 47,48 ).

A physical basis for understanding the abundance variations was provided by applying the same theory of selfgenerated waves that clarified the streaming-limited intensities and spectra. $\mathrm{Ng}$ et al. $(24,25)$ modified the earlier calculations of particle transport through self-generated waves (23) by injecting power-law energy spectra with coronal abundances at the position of the shock as it moved out toward Earth. Wave generation and pitch-angle scattering of the ions was followed in full detail in space and time. Abundance variations from this theory $(24,25)$ are shown in Figure 5 for conditions similar to those of the 1998 April 20 west-limb event.

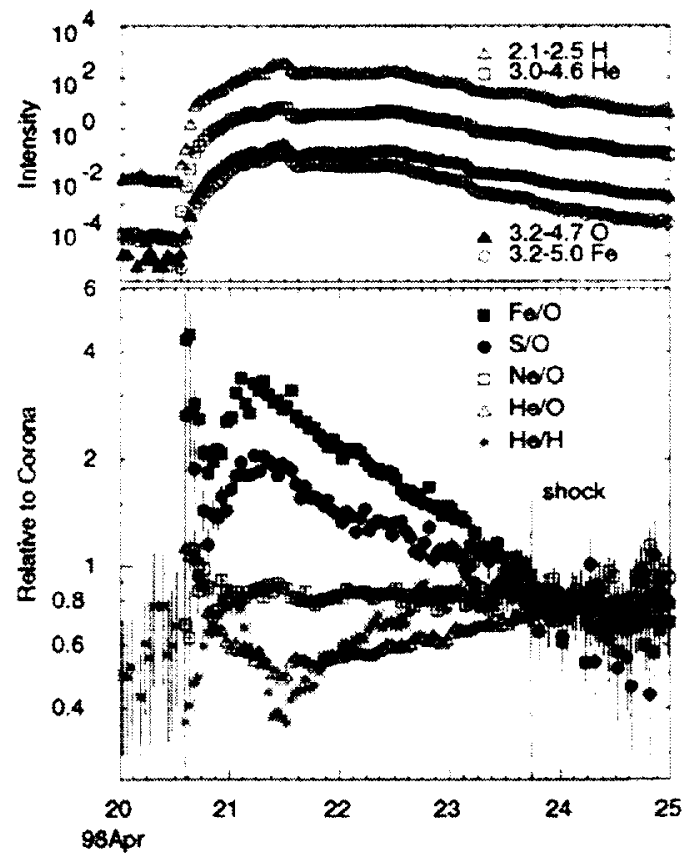

FIGURE 4. Selected intensities and abundance ratios, relative to coronal, are shown for the 1998 April 20 event. Abundances respond to the varying wave specura ahead of the shock. 
The behavior of the variations in Figure 5 may be understood qualitatively as follows. Barly in the event. Fe is scattered slightly less than $O$ of the same velocity, even though the scattering mean frec paths. $\lambda_{i}$, of both species are $-1 \mathrm{AC}$ in the presumed Kolmogorov, $k^{.5 / 3}$, background wave spectrum. Hence, ic arrives first and Fe/O begins high but falls as more $O$ arrives. As wave growth increases with timx. $O$ is preferentially trapped near the shock and $\mathrm{Fe} / \mathrm{O}$ at $1 \mathrm{AL}$ rises. To ohtain the final decease in $\mathrm{F}: \mathrm{O}$ and the greater decrease at higher energy. we must decrease the shock compression ratio with time. as might be quite appropriate for an event near the west limb of the Sun. For a shock that remains stronger. $\mathrm{Fe} / \mathrm{O}$ would remain high and its value would increase with energy as is seen in other events.

The $\mathrm{He} / \mathrm{H}$ ratio in Figure 5 is especially interesting. Its behavior is almost a mirror image of that of $\mathrm{Fe} / \mathrm{O}$. However, early in the event, before the first protons to arrive have had time to grow waves, one might expect $\mathrm{He} / \mathrm{H}$ to behave like $\mathrm{Fe} / \mathrm{O}$. Both involve the ratio of high-rigidity/low-rigidity species at the same velocity; both would respond similarly to a Kolmogorov wave spectrum. The secret lies in the fact that $\mathrm{He}$ resonates with waves created by protons of twice its velocity. These waves are created prior to the arrival of the He and scatter the $2.1 \mathrm{MeV} / \mathrm{amu} \mathrm{He}$ while the $2.1 \mathrm{MeV}$ protons have yet to generate resonant waves. Thus, in large events, with substantial wave growth. He/H can rise initially; in smaller events, the ambient power-law wave spectra will produce an initial decline in both $\mathrm{Fe} / \mathrm{O}$ and $\mathrm{He} / \mathrm{H}$. The energy region of the initial rise in $\mathrm{He} / \mathrm{H}$ (and other species) can expand to higher energies in larger, more intense events $(24,25)$. The physical origin of the initial rise or fall in $\mathrm{He} / \mathrm{H}$ has been a mystery to observers for the last 20 years $(50)$.

With the above discussion. we also begin to understand the poor correlation between $\mathrm{He} / \mathrm{H}$ and $\mathrm{Fe} / \mathrm{O}$ on an event-averaged basis. Furthermore, at its present level. the theory only considers differential transport of ions, not differential acceleration. If $\mathrm{Fe} / \mathrm{O}$ is enhanced in one place, it must be reduced in another; the spatial integral of all species shoukd yield coronal abundances. Thus, it becomes clearer why averaging abundances over many events, Fe-rich and Fe-poor, recaptures coronal abundances and produces the familiar plot of averaged abundance ratios as a function of FIP. 
Based on recent abundance measurements at significantly higher energy on ACE/SIS. Cohen et al. (4) have suggested that while fie-poor events may come from shock acceleration. Fe-rich events come from flares, or worse, some other unknown mechanism. I woukd suggest that the true problem here is that the measurements were made at an energy where large abundance variations are known to exist. Tylka $e t$ al.

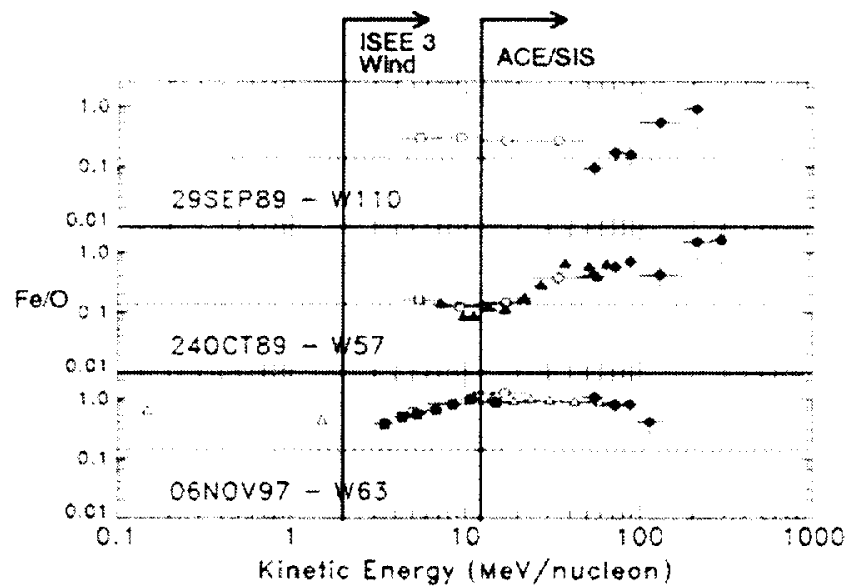

FIGURE 6. Energy thresholds of various instruments are superposed on Fe/O data from Tylka et al. (44). The gradual events in 1989 have $Q_{F e}-14$ up to $600 \mathrm{MeV} / \mathrm{amu}(46): Q_{F}$ for the 1997 Nov, 6 was discussed and is shown in Figure 1. (44) previously found enhancements of $\mathrm{Fe}$ extending to much higher energies, even in the same events of September and Octoher of 1989 where they found $Q_{\mathrm{Fe}} \sim 14$ from $200-600 \mathrm{MeV} / \mathrm{amu}$ (46). These $\mathrm{Fe} / \mathrm{O}$ ratios are shown in Figure 6 together with the regions of energy coverage of the ACE/SIS. Wind, and ISEE-3 instruments. Furthermore, Mazur $(19,21)$ studied the energy dependence of abundances in 10 large SEP events. He found large abundance variations at high energies while, at low energies, the event-to-event abundance variations become smaller and the mean abundances approached those of the corona and solar wind. All of the abundance measurements of Reames $(28,31,32$ ) were made using ISEE-3 data. At a few MeV/amu the resolution of impulsive and gradual events is clear (36), above $15 \mathrm{MeV} / \mathrm{amu}$, it is not. If we examine the 4 events studicd by Cohen et al. (4), the abundances seen on the Wind spacecraft at $\sim 2 \mathrm{MeV} / \mathrm{amu}$. in the same events, are less dramatic and are consistent with the historic values for gradual events. The high-energy abundances are also consistent with observations during the last two solar cycles; unfortunately, Cohen et al. neither discuss nor reference this earlier work. Ionization-state measurements confirm that the large Fe-rich SEP events are from the same CME-driven shock sources as are the Fe-poor ones. Therefore, it is appropriate to average over all large SEP events to obtain abundances. The fact that this averaging directly produces coronal abundances is no accident.

\section{Angular Distributions}

Another prediction of the $\mathrm{Ng}(24,25)$ model is that wave generation in sufficiently large SEP events rapidly reduces scattering mean free paths, not only at the shock, but also out as far as 1 AU. Figure 7 compares intensity-time profiles and magneticsectored intensities of $\mathrm{H}$ and $\mathrm{He}$ in "large" and "small" events seen by the Wind spacecraft. The sectored data are produced by binning individual ions identified onboard into 16 sectors spanning $360^{\circ}$ relative to the direction of the magnetic field as the 


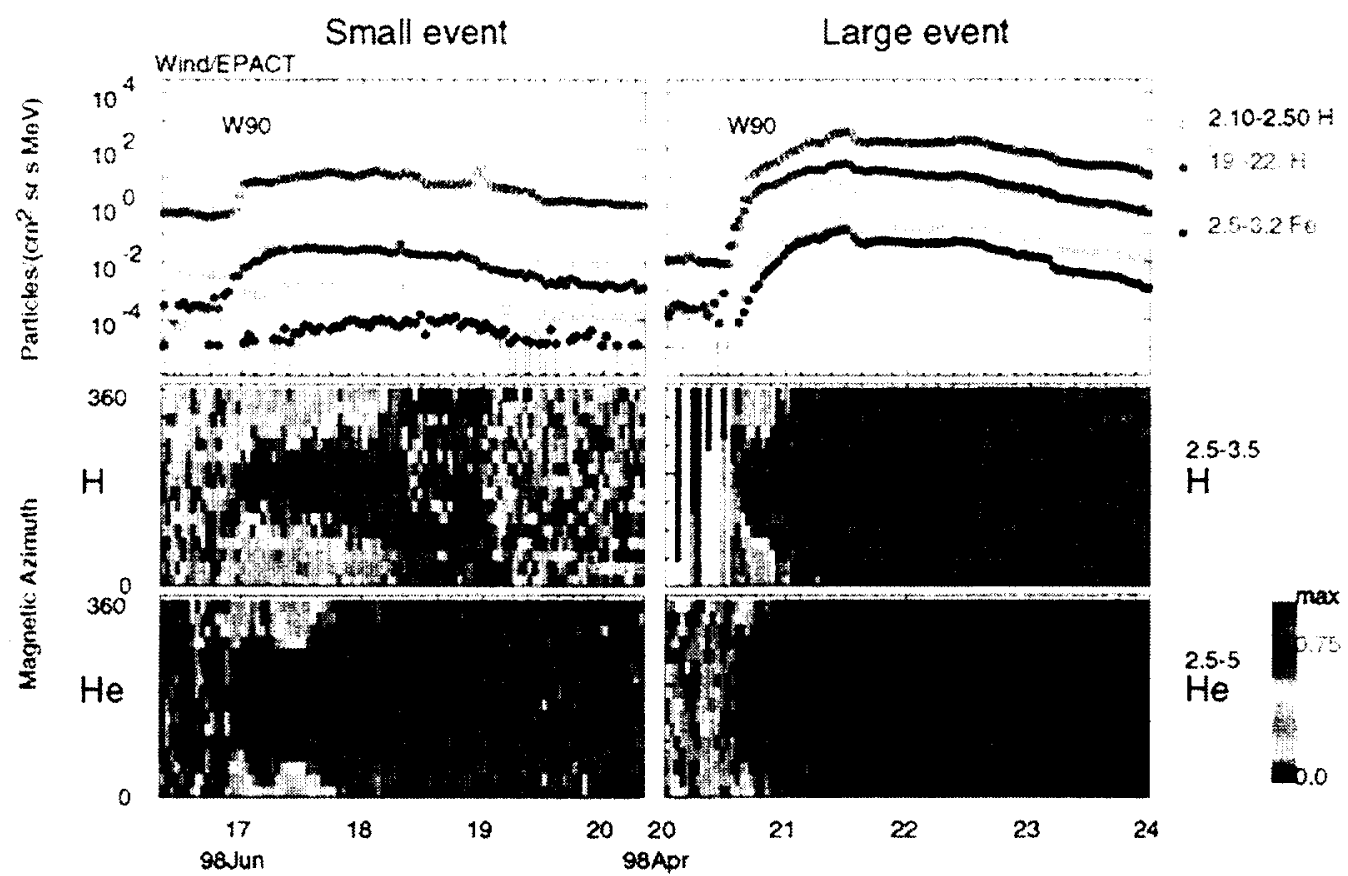

FIGURE 7. Intensities and angular distributions relative to the magnetic field are for compared for "small" and "large" SEP events. Wave generation in the large events rapidly reduces streaming along the field and isotropizes the particles.

spacecraft spins with spin axis normal to the ecliptic plane. The magnetic azimuth is updated every 3 seconds onboard the spacecraft and high-speed onboard processing can accommodate all particles that enter the EPACT/LEMT telescope. Figure 7 shows initial particle tlow centered on $180^{\circ}$ to the field direction early in both events. However. this flow persists for more than a day in the small 1998 June 16 event, but the particles rapidly isotropize within hours in the 1998 April 20 event. Peak proton intensities differ by a factor of $\sim 100$ in the two events at $\sim 2 \mathrm{MeV}$; evolution of the scattering mean free paths for the April event simulation are show by $\mathrm{Ng}$ et al. (24).

This wave-generated scattering in large SEP events explains another historic controversy. Early measurements, which were limited to large events by the instrument sensitivity. found scattering mean free paths, $\lambda<0.1 \mathrm{AU}$, while later observations of small impulsive-flare events often found $\lambda>1 \mathrm{AU}$ (see review 32). Could the interplanetary medium distinguish the particles from different events and scatter them differently? Ironically, the answer is yes for events that occur at different times. Protons from large events rapidly modify the interplanetary medium; they are no longer "test paricles" that probe pre-existing turbulence.

\section{The "Knee" of the SEP Spectrum}

After a sufficiently long time. the spectrum of shock-accelerated particles will begin to approach an equilibrium power-law form extending to arbitrarily high energies with an index that depends only on the shock compression ratio (8). For real shocks. how- 
ever. this equilibrium is not attained at high energies where the scattering mean free path and the acceleration time may be long.

lovell et al. (16) showed a proton energy spectrum extending from 1 $\mathrm{MeV}$ to $10 \mathrm{GeV}$ for the large SEP event of 1989 September 29 as shown in Figure 8. This event is a wellstudied shock event from a source just beyond the west limb of the sun; it is an event for which $Q_{\mathrm{Fe}}-14$ was observed in the 200-600 MeV/amu region (46). The spectral region from 1 to $10 \mathrm{GeV}$, shown as shaded in Figure 8, was obtained by fitting ground-level neutronmonitor measurements from many stations. The fitted curves for $\mathrm{H}$ and $\mathrm{He}$ in the figure come from the shock theory of Ellison and Ramaty (5), which has the form of a power law times an exponential in energy/nucleon. The exponential rollover in this theory, which scales as $Q / A$. was originally used to explain differences in electron. proton and He spectra in large SEP events.

In smaller events, the spectral "knee" occurs at much lower energies. Element abundances near the knee in the 1998 April 20 event are shown in Figure 9 (45). In this event, the e-folding constant, $E_{0}$ in the exponential rollover scales like $Q / A$ times energy/nucleon.

Comparison of Figures 8 and 9 raises an obvious question. Why does the proton spectral knee occur at $-20 \mathrm{MeV}$ in one event and at $\sim 1 \mathrm{GeV}$ in another? This large difference can have little to do with ambient scattering conditions for high-energy protons prior to the event. It may occur because one event has a faster shock, higher proton intensities. and much greater wave growth that can

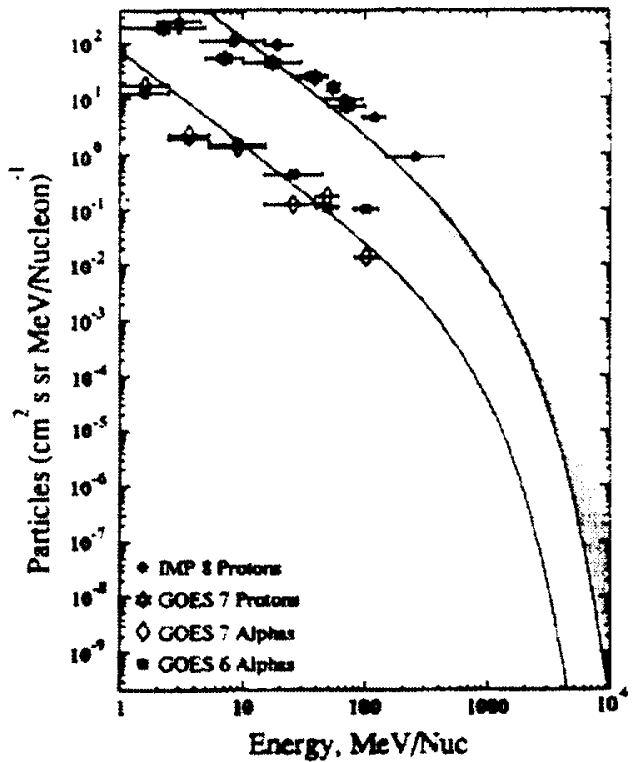

Figure 8. Energy spectra in the 1989 September 30 event as measured on IMP 8, GOES, and by ground-level neutron monitors (shaded region) (16). Fitted curves are from shock theory (5).

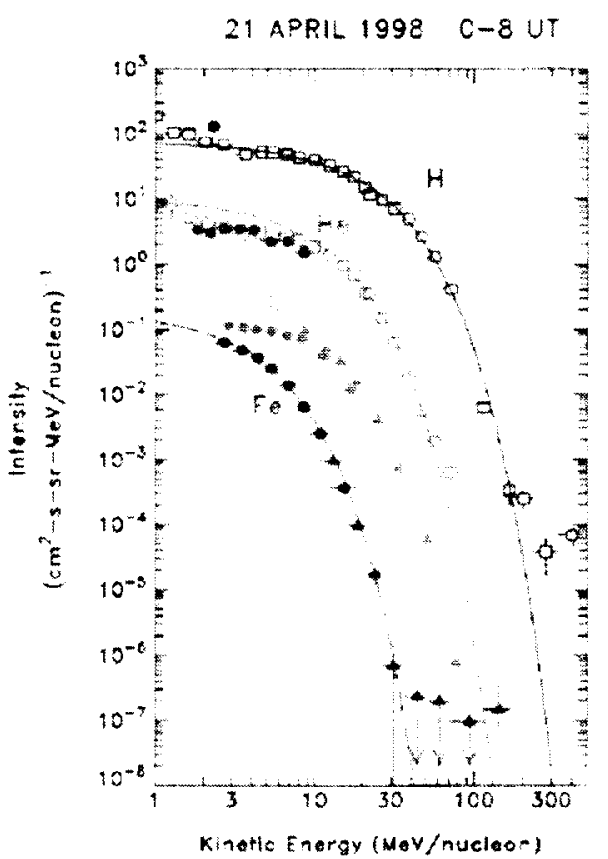

FIGURE 9. Energy spectra of various ion species in the 1998 April 20 event (45) observed by IMP 8, WIND, and ACE. 
persist to much higher energies. The position of the knee is a consequence of the strength of the shock and the intensity of proton-generated waves.

\section{CONCLUSIONS}

New measurements of particle intensities, ionization states, spectra and abundances. together with new observation of CMLs, continue to support the premise that acceleration in large SEP events occurs at CME-driven shock waves. Of course, observations in some energy regions are too highly variable to be helpful in resolving these gradual events from impulsive-flare related events.

Many of the features we observe in the large SEP events are a consequence of proton-generated waves near shocks. These include the following:

1) Streaming-limited intensities early in large events (23, 24, 26, 37, 32).

2) Flattened low-energy spectra in large events $(24,32,47)$.

3) Systematic time variations in abundances $(31,32,24,25,47,48)$.

4) Abundance variations that average to coronal (FIP-dependent) values (32).

5) $\mathrm{He} / \mathrm{H}$ uncorrelated with $\mathrm{Fe} / \mathrm{O}$; breakdown of the power law of abundances is. $Q / A$ resulting from non-Komolgorov wave spectra $(24,25,32,47)$.

6) Initial rise in $\mathrm{He} / \mathrm{H}$ in large events $(24,25,47,32)$.

7) Rapid onset of isotropy in large events, even at $1 \mathrm{AU}$.

8) Large variations in the energy of the spectral "knee" $(32,45)$.

Amazingly, all these results arise from wave energy that is less than a few percent of the energy in SEPS. This list has come from the close collaboration of observations and theory that is as uncommon as it is highly rewarding. Not only has the new theory been able to explain historic questions that have lain dormant up to 20 years. It has also predicted new behavior that was subsequently observed.

CME-driven shock waves provide a richly diverse laboratory in which to study the physics of particle acceleration. Shock acceleration occurs widely at sites throughout the heliosphere. However. only in SEP events can we examine that process for hundreds of events under such a wide range of initial conditions. We are only beginning to organize the diversity we see.

\section{ACKNOWLEDGEMENTS}

1 would like to thank Allan Tylka for providing the data for Figures 6 and 9 and Jenny Lovell for Figure 8. I am indebted to Steve Kahler, Chee Ng, and Allan Tylka for their helpful comments on this manuscript.

\section{REFERENCES}

1. Barghouty, A. F., and Mewaldt, R. A., Astrophys. J. (Letters), 520, L127 (1999).

2. Bieber, J. W. Wanner, W., and Matthaeus, W. H., J. Geophys. Res. 101. 2511 (1996).

3. Breneman, H. H., and Stone, E. C., Astrophys. J. (Letters) 299. L57 (1985).

4. Cohen, C. M. S., ProC. 26 1 ICRC (Salt Lake City) 6. 244 (1999); also Geophys. Res. Lett. $26,2697$.

5. Ellison, D., and Ramaty, R., Astrophys. J. 298, 40X) (1985)

6. Fisk, L. A., in Solar System Plasma Physics, Vol. 1, edited by E. N. Parker, C. F. Kennel, 1. J. Lanzerotti (Amsterdan: North Holland), p. 177 (1979). 
7. Gosling. J. T., J. (ieophys. Res. 98, 18949 (1993).

8. Junes. F. C... and Ellison. D. L.. Space Sci. Revs 58. $25911991 \%$

9. Kahler, S. W.. Ann. Rev. Astron. Astrophys. 30, 113 (1992).

10. Kuhler. S. W.. Astrophs. J. 428, 837 (1994).

11. Kahler, S. W. co al. J. Geophys. Res. 89, $9683(1984)$.

12. Kennel, C. F. et al. J. Geophys Res 91, 11917 (1986).

13. L.ex. M. A.. J. (jeophss. Res. 88, 6109 (1983).

14. L.e. M. A., Proc, $22^{\text {sid }}$ Int Cos. Ray Conf., (Dublin) 5.293 (1991).

15. Lee, M. A.. in: Coronal Mass Ejections, edited by N. Crooker, J. A. Jocelyn, J. Feymman, Geophys. Monograph 99. (AGI press) p. 227 (1997).

16. Lovell, J. L., Duldig, M. L., Humble, J. E., J. Geophys. Res. 103, 23,733 (1998).

17. Luhn. A.. Klecker, B., Hovestadt. D., and Möbius, E., Astrophys. J. 317. 951 (1987).

18. Mobius, E., M., et al., Geophws. Res. Lett. 26. $145(1999)$.

19. Mazur. J. E.. Univ. of Maryland thesis (1991).

20. Mazur. J. E., et al.. Geophys. Res. Lett. 26, 173 (1999).

21. Mazur, J.E.. Mason. G.M., Klecker, B., and McGuire. R.E., Astrophys. J. 401, 398 (1992).

22. Meyer, J. P., Astrophys. J. Suppl. 57. 151 (1985).

23. Ng. C. K., and Reames, D. V. Astrophys. J. 424, 1032 (1994).

24. Ng. C. K., Reames, D. V., and Tylka. A. J., Geophys. Res. Lett. 26, 2145 (1999).

25. Ng, C. K.. Reames. D. V., and Tylka, A. J., Proc. 26 ${ }^{\text {th }}$ ICRC (Sall Lake City) 6, 151 (1999).

26. Reames. D. V.. Astrophys. J. (Letters), 358, L63 (1990)a).

27. Reames. D. V., Astrophys. J. Suppl. 73, 235 (1990b).

28. Reames. D. V.. Adr. Space Res. 15 (7), 41 (1995a).

29. Reames, D. V., Revs. Geophys. (Suppl.) 33, (U. S. National Repont to the IUGG), 585 (1995b).

30. Reames. D. V. in: Coronal Mass Ejections, edited by N. Crooker, J. A. Jocelyn. J. Feymman, Geophys. Monograph 99. (AGU press) p. 217 (1997).

31. Reames, D. V.. Space Science Revs. 85, 327 (1998).

32. Reames, D. V.. Space Science Revs., in press (Dec 1999).

33. Reames, D. V., Barbier. L. M., and Ng. C. K., Astrophys. J. 466, 473 (1996).

34. Reames, D. V., Kahler, S. W., and Ng, C. K., Astrophys. J. 491, 414 (1997b).

35. Reames, D. V., Kahler, S. W, and Richardson, I. G., Geophys. Res. Lett., submitted (1999).

36. Reames, D. V., Meyer, J. P., and von Rosenvinge, T. T., Astrophys. J. Suppl., 90, 649 (1994).

37. Reames. D. V., and Ng. C. K., Astrophys. J. 504, 1002 (1998).

38. Reames, D. V., Ng, C. K., and Tylka, A. J., Geophys. Res. Lett. submitted (1999).

39. Roth, I., and Temerin. M., Astrophys. J. 477, 940 (1997).

40. Ruffolo, D., Astrophys. J. (Letters) 481, L119 (1997).

41. Stix, T. H., The Theon of Plasma Waves (New York: McGraw-Hill) (1962).

42. Tan, L. C., Mason, G. M., Gloeckler, G., and Ipavich, F. M., J. Geophys. Res. 94, 6554 (1989).

43. Tsurutani, B. T., and Gonzalez, W. D., Planet. Space Sci. 35, 405 (1987).

44. Tylka, A. J., Dietrich, W. F., and Boberg. P. R., Proc. $25^{\text {th }}$ Int. Cos. Ray Conf., (Durban) 1, 101 (1997); also Tylka. A. J., and Dietrich, W. F., Radiation Meas. 30-3, 345 (1999).

45. Tylka, A. J., Dietrich, W. F., Lopate and Reames, D. V., Proc. 26 Int. Cos. Ray Conf. (Salt Lake City) 6, 67 (1999): also Tylka. A. J., et al., in preparation (1999).

46. Tylka, A. J., Boberg, P. R., Adams, J. H., Jr., Beahm, L. P., Dietrich, W. F., and Kleis, T., Astrophws. J. (Letters) 444. L109 (1995).

47. Tylka, A. J., Reames, D. V., and Ng, C. K, Geophys. Res. Lett. 26, 2141 (1999).

48. Tylka, A. J., Reames, D. V., and Ng, C. K, Proc. 26 $6^{\text {th }}$ ICRC (Salt Lake City) 6, 135 (1999).

49. Vinas, A. F., Goldstein, M. L., and Acuña, M. H., J. Geophys. Res,. 89, 3762 (1984).

50. Witte. M. Wibberenz, G., Kunow, H., and Muller-Mellin, R., Proc. $16^{\text {th }}$ ICRC, (Kyoto) 5. 79 (1979). 